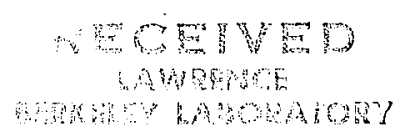

\title{
Noy 179078
}

\section{TWO-WEEK LOAN COPY}

This is a Library Circulating Copy which may be borrowed for two weeks. For a personal retention copy, call Tech. Info. Dívision, Ext. 6782

Free Convective Laminar Flow Within The Trombe Wall Channel

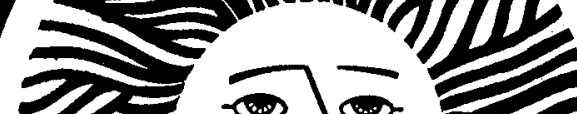
$\infty$ 


\section{LEGAL NOTICE}

This report was prepared as an account of work sponsored by the United States Government. Neither the United States nor the Department of Energy, nor any of their employees, nor any of their contractors, subcontractors, or their employees, makes any warranty, express or implied, or assumes any legal liability or responsibility for the accuracy, completeness or usefulness of any information, apparatus, product or process disclosed, or represents that its use would not infringe privately owned rights.

Printed in the United States of America

Available from

National Technical Information Service

U. S. Department of Commerce

5285 Port Royal Road

Springfield, VA 22161

Price: Printed Copy, \$ 4.50 Domestic; \$ 9.00 Foreign

Microfiche, $\$ 3.00$ Domestic; $\$ 4.50$ Foreign 
FREE CONVECTIVE LAMINAR FLOW WITHIN THE TROMBE WALL CHANNEL

H. Akbari and T.R. Borgers

\author{
Energy and Environment Division \\ Lawrence Berkeley Laboratory \\ University of California \\ Berkeley, CA 94720
}

This work has been supported by the Solar Heating and Cooling Research and Development Branch, Office of Conservation and Solar Applications, U.S. Department of Energy. 


\title{
FREE CONVECTIVE LAMINAR FLOW WITHIN THE TROMBE WALL CHANNEL
}

\author{
H. Akbari and T.R. Borgers \\ Energy and Environment Division \\ Lawrence Berkeley Laboratory \\ University of California \\ Berkeley, CA 94720
}

\begin{abstract}
Free convective laminar heat transfer between the channel surfaces of the Trombe wall has been investigated. This study considered the velocity profiles normal and parallel to the direction of fluid flow, the pressure drop due to flow acceleration at the channel entrance, and the effect of a variety of dissimilar but uniform channel surface temperatures for a wide range of flow rates.

A finite difference procedure was used to solve the governing equations in dimensionless form using air as the fluid. After comparison with available experimental data, results have been reduced, and several correlations developed to enable important performance characteristics to be estimated given the channel thickness, height, and surface temperatures.
\end{abstract}




\section{INTRODUCTION}

The convective contribution to heating by a Trombe wall involves several geometric and thermal parameters. During a typical diurnal cycle, experimental evidence seems to indicate that laminar flow exists for a smaller fraction of the time that the wall acquires heat; and that turbulent flow becomes dominant as the channel surfaces reach higher temperatures [1]. Several important performance characteristics of the Trombe wall have been measured by Balcomb, et al [2].

The problem of laminar natural-convective flow between parallel plates was first studied by Elenbass [3]. Experimental work was also done by Kobayashi and Fujimoto [4], Siegel and Norris [5], Currie and Newman [6] and Aihara [7]. Bodoia and Osterle applied finite difference techniques in their study [8]. Engle and Mue11er [9] attacked the problem by the integral method. The finite difference method was later employed in the studies by Miyatake and Fujii [10], and by Aihara [11]. These investigations greatly improved the understanding of the effects of the inlet velocity profiles and the nature of the fluid pressures within the channe1.

The broad spectrum of temperature differences experienced during the normal operation of the Trombe wall results in low to moderately high volume flow rates. Thus, the purpose of this study is to extend the previous work to Trombe wall dimensions, to regions of lower flow rates and unequal surface temperatures and to provide the results in an easily used form. This phase is restricted to the study of laminar flow between two parallel plates, each of which is at some effective uniform temperature, for a wide range of flow rates. For very low 
volumetric flow rates, the performance characteristics can apparently be estimated using single plate formulations. Several correlations have been developed from the computed results which may be useful in building design.

\section{THEORY}

The geometry of the flow problem considered is shown in Figure 1. Two parallel, infinitely wide vertical plates, perfectly insulated on the outside, have their bases in contact with a calm fluid at temperature $T_{0}$. The temperatures of the plates are constant and uniform at values $T_{g}$ and $T_{W}$ both of which are greater than $T_{0}$.

Fluid properties, except density, are assumed to be independent of temperature. The decrease of fluid density upon heating is solely responsible for buoyancy forces which induce upward flow in the channel. The flow is assumed to be steady, laminar, compressible, and is governed by the equations of continuity, momentum and energy, which simplify to:

$$
\begin{aligned}
& \partial u / \partial x+\partial v / \partial y=0 \\
& u \partial u / \partial x+v \partial u / \partial y=-\rho^{-1} \partial\left(p-p_{\infty}\right) / \partial x \\
& +v\left(\partial^{2} u / \partial x^{2}+\partial^{2} u / \partial y^{2}\right)+g \beta\left(T-T_{0}\right) \\
& u \partial v / \partial x+v \partial v / \partial y=-\rho^{-1} \partial\left(p-p_{\infty}\right) / \partial y \\
& +v\left(\partial^{2} v / \partial x^{2}+\partial^{2} v / \partial y^{2}\right) \\
& u \partial T / \partial x+v \partial T / \partial y=v \operatorname{Pr}^{-1}\left(\partial^{2} T / \partial x^{2}+\partial^{2} T / \partial y^{2}\right)
\end{aligned}
$$

The pressure within the channel, p, will be less than the hydrostatis pressure, $p_{\infty}$, outside the channel at the same elevation. The difference between them, $\left(p-p_{\infty}\right)$, is the pressure defect. 
These relations may be restated in dimensionless form using variables in Nomenclature. Assuming that $\mathrm{Gr} \gg 1$, the governing equations reduce to:

$$
\begin{aligned}
& \partial U / \partial X+\partial V / \partial Y=0 \\
& U \partial U / \partial X+V \partial U / \partial Y=-\partial P / \partial X+\partial^{2} U / \partial Y^{2}+\theta \\
& \partial P / \partial Y=0
\end{aligned}
$$

and

$$
\mathrm{U} \partial \theta / \partial X+V \partial \theta / \partial Y=\operatorname{Pr}^{-1} \partial^{2} \theta / \partial Y^{2}
$$

The boundary conditions are:

$$
\begin{aligned}
& X=0 \text { and for } 0 \leqslant Y \leqslant 1 ; \\
& U=U_{0}, V=0, \theta=0, P=P_{0}
\end{aligned}
$$

when

$$
\begin{aligned}
0 \leqslant X \leqslant L \quad \text { and } \quad Y=0 ; \\
U=0, \quad V=0, \theta=\theta_{g} \\
0 \leqslant X \leqslant L \quad \text { and } Y=1 ; \\
U=0, \quad V=0, \theta=\theta_{W} \\
\text { at } \quad X=L ; P=0
\end{aligned}
$$

$P_{0}$, the pressure defect at the inlet, is a function of $U_{0}$, the inlet velocity profile, which should be determined by the analys is of the "free-boundary" problem. Because of the effort to obtain information on the behavior of the flow at low volumetric flow rates, a constant inlet velocity profile, and an inlet pressure defect value of $-Q^{2} / 2$ were selected. This term in the pressure defect at the entrance represents only the dynamic contribution. Other terms, which are unknown and not included, are the effects of eddys and turbulence, and would result in a somewhat more negative pressure defect. However, 
because a great variety of inlet and exit configurations are possible in the practical construction of a Trombe wall, these assumptions are judged to be appropriate at this time. Experiments, now in the planning stages, are addressing these issues.

The dimensionless, volumetric flow rate is

$$
Q=\bar{u} b v^{-1} G r^{-1}=\int_{0}^{1} U d Y
$$

which must remain constant throughout the channel. The dimensionless rate of heat absorption by the fluid up to a particular elevation $X$ is

$$
\begin{aligned}
H_{x} & =\nu^{-1} G r^{-1}\left(T_{m}-T_{0}\right)^{-1} \int_{0}^{b} u\left(T-T_{0}\right) d y \\
& =\int_{0}^{T} U \theta d Y
\end{aligned}
$$

The mixed-mean fluid temperature at a particular elevation $X$ in dimensionless form is

$$
\bar{\theta}_{f}=H_{x} / Q \text {. }
$$

The local Nusselt numbers at the glass and wall surfaces are respectively,

$$
\begin{aligned}
& N u_{x, g}=h_{x, g} \cdot b / k=(\partial \theta / \partial Y)_{x, g} \\
& N u_{x, w}=h_{x, w} \cdot b / k=(\partial \theta / \partial Y)_{x, w}
\end{aligned}
$$

and the total local Nusselt number is expressed as $N u_{x, t}=N u_{x, g}+$ $\mathrm{Nu}_{\mathrm{x}, \mathrm{w}^{\circ}}$

In addition we may define a total average Nusselt number as

$$
\overline{\mathrm{Nu}}_{\mathrm{t}}=\mathrm{H}_{\mathrm{L}} \cdot \mathrm{Pr} / \mathrm{L}
$$


where $H_{L}$ is the rate of total heat absorption by the fluid at the exit elevation, $X=L$.

\section{FINITE-DIFFERENCE FORMULATION AND THE METHOD OF SOLUTION}

The equations were solved using a forward-marching, line-by-line implicit finite-difference technique permitting iterations on each new line similar to that described by Aihara [11].

A rectangular grid, across the channel width, see Figure 2, is used to establish the increments of the finite-difference approximations to eqns. (5), (6), and (8). In general, the expressions are of the form

$$
\begin{aligned}
& U \partial \eta / \partial X \doteq U_{j+1, k}^{*}\left[n_{j+1, k}-n_{j, k}\right](\Delta X)^{-1} \\
& V \partial \eta / \partial Y \doteq V_{j+1, k}^{*}\left[n_{j+1, k+1}-n_{j+1, k-1}\right](2 \Delta Y)^{-1} \\
& \partial^{2} n / \partial Y^{2} \doteq\left[\eta_{j+1, k+1}-2 \eta_{j+1, k}+\eta_{j+1, k-1}\right](\Delta Y)^{-2}
\end{aligned}
$$

where $n$ may be either $U$ or $\theta$. The superscript *, refers to the values of $U$ or $V$ from the previous iteration on the $j+1$ line.

Upon substituting the finite-difference approximations into eqns. (5), (6) and (8), then making use of the boundary conditions, a set of (K-2) Tinear equations for $U_{j+1, k}, \theta_{j+1, k}$ and $V_{j+1, k}$ are obtained. First the energy equation is solved, providing a temperature $\theta_{j+1, k}$, which is used in the solution of the momentum equation. The $U$ velocity which results is used to calculate a new $V$ velocity based upon the continuity equation.

The energy, momentum, and continuity equations in matrix form are: 


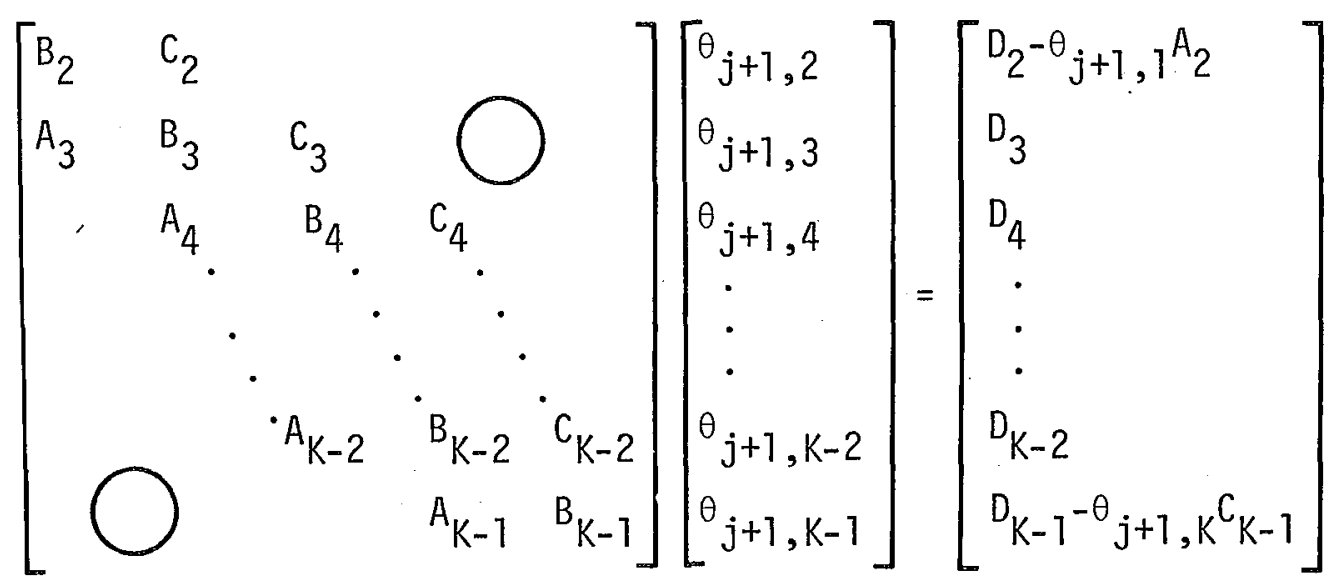

where

$$
\begin{aligned}
& A_{k}=-V_{j+1, k}^{*}(2 \Delta Y)^{-1}-\operatorname{Pr}^{-1}(\Delta Y)^{-2} \\
& B_{k}=U_{j+1, k}^{*}(\Delta X)^{-1}+2 \operatorname{Pr}^{-1}(\Delta Y)^{-2} \\
& C_{k}=V_{j+1, k}^{*}(2 \Delta Y)^{-1}-\operatorname{Pr}^{-1}(\Delta Y)^{-2} \\
& D_{k}=U_{j+1, k}^{*}{ }_{j, k}(\Delta X)^{-1}
\end{aligned}
$$

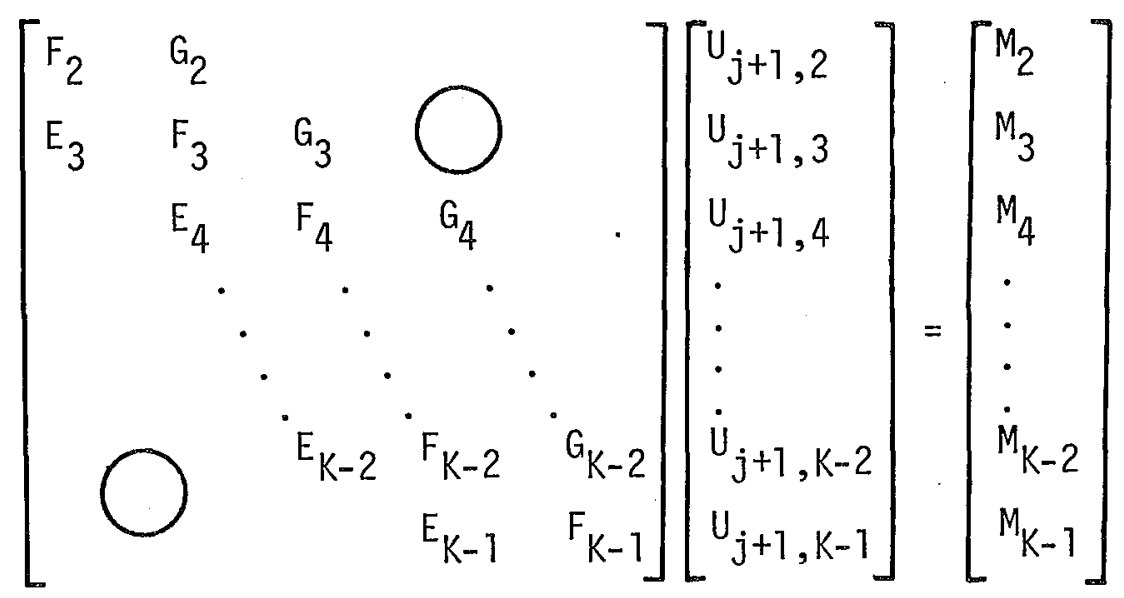

where

$$
\begin{aligned}
E_{k}=-V_{j+1, k}^{*}(2 \Delta Y)^{-1}-(\Delta Y)^{-2} ; F_{k} & =U_{j+1, k}^{*}(\Delta X)^{-1}+2(\Delta Y)^{-2} \\
G_{k}=V_{j+1, k}^{*}(2 \Delta Y)^{-1}-(\Delta Y)^{-2} ; M_{k} & =U_{j+1, k}^{*} U_{j, k}(\Delta X)^{-1} \\
& -(d P / d X)_{j+1}+\theta_{j+1, k}
\end{aligned}
$$




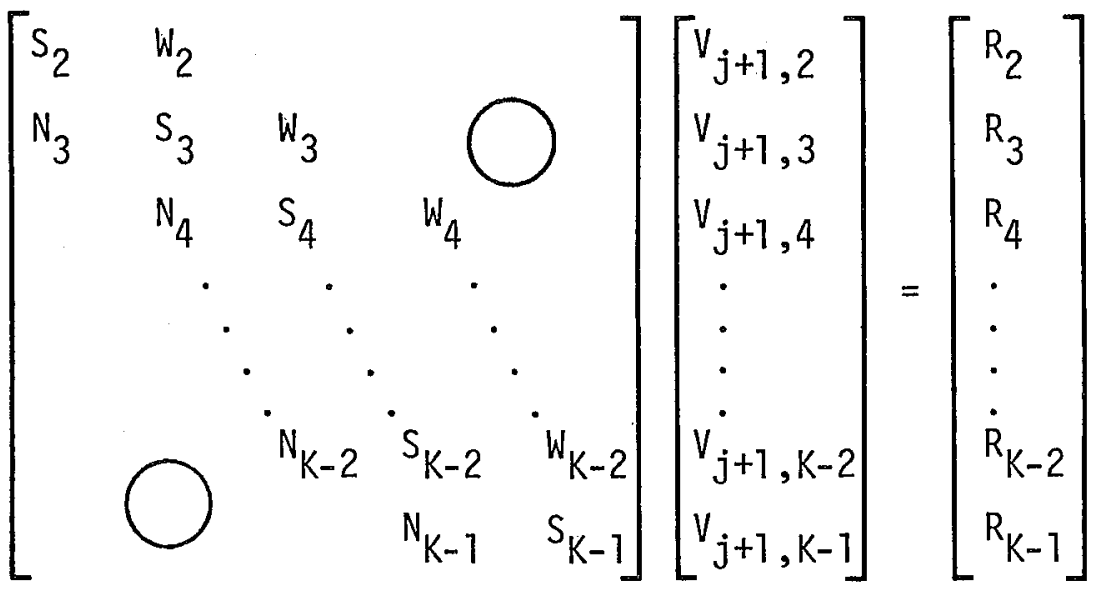

$$
\begin{aligned}
& \text { where } N_{k}=(1-Y) ; S_{k}=-1 ; W_{k}=Y \\
& \begin{aligned}
R_{k} & =\Delta Y(2 \Delta X)^{-1}\left[\left(U_{j+1, k}-U_{j, k}+U_{j+1, k-1}-U_{j, k-1}\right)(1-Y)\right. \\
& \left.-\left(U_{j+1, k}-U_{j, k}+U_{j+1, k+1}-U_{j, k+1}\right) Y\right] .
\end{aligned}
\end{aligned}
$$

The solutions are quickly obtained using Gaussian elimination with the starting values of $V_{j+1, k}^{*}$ and $U_{j+1, k}^{*}$ taken to be converged values of the previous row, namely $V_{j, k}$ and $U_{j, k}$. A discussion of the pressure gradient, $(d P / d X)_{j+1}$, is presented in Appendix 1 .

The calculation procedure follows quite closely that presented by Aihara [1]]. The inlet velocity profile, the volumetric flow rate, and the thermal boundary conditions are chosen. At the next row $(j+1=2)$ the governing equations are solved to determine the new values of $U, V$, and $\theta$ for the $j+1$ row. A new volumetric flow rate $Q_{j+1}$ is calculated from the new $U$ velocity profile using eqn. (13) by the trapezoidal rule approximation. This procedure is repeated with a corrected pressure gradient until

$$
\frac{Q_{j+1}-Q}{Q} \leqslant \varepsilon
$$


where epsilon determines the convergence tolerance. When convergence is obtained, the pressure defect at this row, $P_{j+1}$, is calculated from the latest corrected pressure gradient and the pressure defect from the previous row:

$$
P_{j+1}=P_{j}+\Delta X(d P / d X)_{j+1}
$$

At this time, the local Nusselt numbers, $\mathrm{Nu} \mathrm{x}_{, g}, \mathrm{Nu} \mathrm{x}_{\mathrm{w}}, \mathrm{Nu} \mathrm{x}_{\mathrm{t}}, \mathrm{\text {,therate }}$ of heat absorption by the fluid at this elevation, $H_{x}$, and the mixed mean fluid temperature, $\bar{\theta}_{f}$ are determined.

The $U_{j+1}, V_{j+1}$, and $(d P / d X)_{j+1}$ values are then carried forward to the next higher row, $j+2$, and the process is repeated. This 1 ine-byline advancement up the channel is continued to a channel height at which the pressure defect becomes positive. Quadratic interpolation of the latest pressure defect values is used to estimate the channel height at which the pressure defect vanishes. This channel height, $L$, together with $H_{L}$, the total rate of heat absorption by the leaving fluid, are used to calculate the overall average Nusselt number as given in eqn. (18)

The calculations for which results are presented deal only with a fluid of Prandtl number $=0.7$ (air). Grid sizes were fixed in the $Y$ direction and were allowed to increase with elevation in the $x$ direction as described in Appendix 1. At higher flow rates, convergence was frequently attained by the fifth iteration. At the lower flow rates, near regions of numerical instability, convergence was reached in certain instances only after 20 to 40 iterations. A more complete discussion is presented in Appendix 1. 


\section{DISCUSSION OF RESULTS}

In order to check the validity of the model used in this study, the half channel results of Aihara [11] were duplicated. Emphas is was then directed to the behavior of systems having lower flow rates and plates of unequal temperatures. There is very little pertinent experimental data in the literature to which these results can be compared. Efforts to study the behavior of an experimental system in detail, are in the planning stages. The results of this investigation are therefore presented, fully aware of the need for experimental validation.

The next several paragraphs briefly discuss a few of the important results and trends. Figures 3-5 show details of the $X$ and $Y$ velocity components labeled $U$ and $V$ respectively, and the fluid temperature, $\theta_{f}$, of a high, intermediate and low flow rate for three different relative surface temperatures and at four relative wall elevations.

At high flows, the $U$ velocity profile shows parabolic development soon after entry when the plates are at equal temperatures. As the glass temperature is lowered, progressively more asymmetric $U$ velocity profiles develop.

The $V$ component represents fluid movement across the channel gap caused by a combination of friction and heating. At high flows an initial rapid flow toward the channel center develops. Soon after the initial adjustment, the movement of the fluid to the center is greatly diminished, since the parabolic character of the upward velocity is already quite well established.

As the heating becomes asymmetric, the fluid flow away from the cooler surface is noticeably greater than that from the warmer side 
very soon after entry. A greater flow persists toward the warmer side of the channel as the disparity of plate temperatures is increased.

For higher flows, in the case of symmetric heating, the fluid temperatures, ${ }_{f}$, in the central area of the fluid gradually acquire a higher value as the flow proceeds up the channel. The coolest portion of the fluid in this case is the fastest moving central layer. As $\theta_{\mathrm{g}}$ is reduced the profile becomes skewed and the fastest moving portion of the fluid is no longer the coolest. At the exit point the cooler surface is not contributing significantly to the heat gain of the fluid. Further reduction of $\theta_{\mathrm{g}}$ shows that the cooler surface is extracting heat from the fluid near the exit of the channel.

For the lower flow rates (Figures 4,5 ), surface friction is still. largely responsible for the initial acceleration of the fluid. Thereafter a distinct indication of the heating effect is noted in $U$ by the more rapidly moving layers of fluid a small distance from the surfaces, and a progressive decrease in the velocity within the central region. As the development proceeds, the velocity of the heated layers increases substantially while the velocity in the cooler central region subsides.

The cross channel flow, $V$, for the lower flow rates shows again the initial surge of fluid away from the surfaces. Untike the higher flow, apparently fluid immediately begins to move toward both the heated surfaces, away from the central region. The extent of the successive decreases in the maxima of the $V$ profiles, as the channel length is traveled, is not as great as in the case of the higher flows, indicating that fluid continues to migrate out of the central area to form the faster upward moving, warmer streams. 
At low flow rates the heating effect is very important early in the channel, and is especially noticeable as the temperature difference between the plates is increased. At the outset, fluid is shifted away from the cooler surface to a greater extent than it is at the warmer surface, indicating that early heating at the warmer surface is able to accelerate fluid in the upward direction. The more asymmetric the heating is, the more completely the flow is dominated by the warmer surface.

The temperature profiles across the channel show much smaller increases in the central region than those at higher flows. The warmer regions of the fluid are the faster moving layers, and at low glass temperatures, the fluid continues to remove heat from the cooler surface to the channel exit.

The pressure defect as a function of the channel height is shown in Figure 6. The behavior shows that at the high flows, the pressure defect rapidly becomes more negative in the first portion of the channel, then reverses and gradually reaches zero which defines the channel exit. The initial decrease is due primarily to the tremendous adjustment of the flow profile as a result of friction with the stationary surfaces. At very low flow rates the behavior becomes very nearly linear soon after entry. It is for this reason that a linear extrapolation of the pressure defect as a function of $X$ is a good approximation at the lower volumetric flows as discussed in Appendix 1.

Figure 7 illustrates the behavior of the local Nusselt numbers for several important situations. Recalling that $N u_{x, t}=\left(N u_{x, g}+N u_{x, w}\right)$, for high flows and low glass temperatures it is seen that near the exit 
point of the fluid the local total Nusselt number becomes less than the local wall Nusselt number, indicating that the fluid is losing a considerable amount of heat through the glass. However, at low flow rates and low glass temperatures, the cooler surface contributes positively to the heat gain of the fluid to the end of the channel.

\section{CORRELATIONS FOR DESIGN PURPOSES}

Ideal7y, the designer could predict the convective performance of the Trombe wall. Simulation programs can be developed, which require as inputs, simply, the wall height, the glazing to wall distance and the glazing type. Wall and inside glazing surface temperatures would be calculated by the program at regular time intervals using a weather data tape. The output would be in the form of an air volumetric flow rate at an average temperature, $\bar{T}$, or simply a heat flux.

In the following paragraphs, attention is directed to the prediction of the laminar convective performance of the Trombe wall. Least squares techniques were used to express useful parameters in a form which can be readily incorporated in a design computation.

of practical interest is the rate of accumulated heat, $\dot{h}_{x}$, by all the fluid in the channel, at and below elevation point $x$ of the channel. The expression used was:

$$
H_{X}=c \cdot x^{\alpha}
$$

where

$$
\begin{aligned}
& \alpha\left(\theta_{g}, Q\right)=a(Q)+b(Q) \theta_{g} \\
& c\left(\theta_{g}, Q\right)=d(Q) \exp \left[e(Q) \theta_{g}\right]
\end{aligned}
$$


and

$$
\begin{aligned}
& a=-2.292- \\
&-0.1513(\log Q)^{3} \\
& b=1.768+ 1.956 \log Q+0.6997(\log Q)^{2} \\
&+0.0814(\log Q)^{3} \\
& d=-2.096- 2.796 \log Q-1.095(\log Q)^{2} \\
&-0.1386(\log Q)^{3} \\
& e=8.961+ 8.807 \log Q+2.948(\log Q)^{2} \\
&+0.3126(\log Q)^{3}
\end{aligned}
$$

The typical behavior of $\mathrm{H}_{X}$ is shown in Figure 8 . Figure 8 also shows an example of the behavior of the rate of total heat absorption, $H_{L}$, by the fluid at the exit elevation, $X=L$, as a function of the glass temperature. This quantity may be calculated from

$$
H_{L}=L \cdot \overline{N u}_{t} / \mathrm{Pr}
$$

The total average Nusse $1 t$ number $\overline{N u}_{t}$ can be expressed as

$$
\overline{N u}_{t}=a+b \theta_{g}
$$

where

$$
\begin{aligned}
a= & -8.605-9.372 \log Q-2.972(\log Q)^{2} \\
& -0.5229(1 \log Q)^{3} \\
b= & 1.547+0.5623 \log Q+1.213(\log Q)^{2} .
\end{aligned}
$$

An example of how the total average Nusselt number varies with $\theta_{\mathrm{g}}$ is also shown in Figure 8.

The method of calculation used in the fundamental program does not contain the total wall height as an input, rather it is determined by such things as the flow rate and the surface temperatures. A designer 
may wish to develop a volumetric flow rate, Q, at a mean glass temperature $\theta_{g}$. The necessary wall height, $L$, is predicted by

$$
L=a \cdot \theta_{g}^{\alpha}
$$

where

$$
\begin{aligned}
& a=10^{\left[1.35+2.834 \log Q+0.2505(\log Q)^{2}\right]} \\
& \alpha=-0.4081+0.3211 \exp (-480.6 Q) \text {. }
\end{aligned}
$$

Predicted and computed total wall heights as a function of $\theta_{g}$ are shown in Figure 8.

More often, a designer may wish to predict a volumetric flow rate, $Q$, using a fixed wall height, $L$, and various glass temperatures, ${ }_{g}$. This can be accomplished by

$$
Q=10^{\left[a+b \log L+c(\log L)^{2}\right]}
$$

where

$$
\begin{aligned}
& a=0.0851\left[1 .-\exp \left(-3.412\left(\theta_{g}-0.4217\right)\right)\right]^{2}-0.920 \\
& b=0.1331+0.6563 \exp \left(-8.521 \theta_{g}\right) \\
& c=-0.0619+0.07125 \exp \left(-6.762 \theta_{g}\right) .
\end{aligned}
$$

Once $Q$ is predicted for a given $\theta_{g}$ and $L$, the total average Nusselt number may be estimated through eqn. (32). The total rate of heat absorption, $H_{L}$, is then easily calculated by eqn. (31). The mixed mean temperature of the fluid at the exit point is obtained from eqn. (15).

The above correlations have been developed using computed results for flow rates of $0.0003 \leqslant Q \leqslant 0.03$ and temperatures of $0.15 \leqslant \theta_{g} \leqslant 1.10$. It should be noted that even though it has not been stated that ${ }_{g}$ may exceed 1.10, the computed results and correlations which were developed 
are equally valid for $0.15 \leqslant \theta_{g} \leqslant 1 / 0.15$ as seen from the definition of $\theta$, in which $T_{m}$ may be the temperature of the hotter surface.

A relatively systematic check has been made of the predicted and computed values over the range of flow rates and temperatures studied. Eqn (33) predicts wall heights which vary no more than $10 \%$ from computed values for $Q<0.02$ and vary no more than approximately $20 \%$ when $Q \geqslant 0.02$. Eqn (31) gives rates of heat absorption which vary less than $10 \%$ from computed values for a $11 Q \leqslant 0.02$ and may vary by approximately $20 \%$ for $Q>0.02$. Eqn (32) gives total average Nusselt numbers which vary from computed values by less than $10 \%$ throughout the range studied. Random checks of eqn (34) give' values of $Q$ which vary from those computed by no more than $10 \%$.

As the volumetric flow rate becomes very sma11, the $x$ direction velocity of the fluid in the central region becomes negligible. This suggests that the problem may be treated as two uncoupled heated vertical plates. The total rate of heat absorption may then be computed as outlined in [12]. Several comparisons of the heat absorption rate have been made using the correlations developed in this study and the uncoupled single plate results at low flow rates; the results agreed to within $10 \%$. This seems to indicate that an extensive computational study directed to lower flow rates in an effort to obtain information on the important performance characteristics of the Trombe wall is unwarranted.

An example of the predictions given by the correlations for free convective Taminar flow is provided in Appendix 2. 
the derivation of which is alluded to by Aihara. If, after the fourth iteration convergence is not reached, a new pressure gradient, $(d P / d X)_{j+1}^{(n=5)}$, is found by linear interpolation of $(d P / d X)_{j+1}^{(n=3)}$, $Q_{j+1}^{i}$, and $(d P / d X)_{j+1}^{(n=1)}, Q_{j+1}^{i-1}$. Thereafter the above process is repeated every other iteration using the latest two values of $Q_{j+1}$.

In regions of low volumetric flow, instances of numerical instability were encountered and convergence was not always achieved. This problem was circumvented to a large degree by regulating the $X$ dimension grid sizes; i.e., as the flow rates were decreased, the initial values of $\Delta \mathrm{X}$ were diminished, and the rate of increase for $\Delta \mathrm{X}$ was regulated with a multiplier, $M$, using the following relation:

$$
\Delta x_{j+1}=M \cdot \Delta x_{j} \cdot
$$

In addition, $d P / d X$ was linearly extrapolated to the next higher row whenever instability was sensed. The normal mode of calculation was resumed immediately thereafter.

Prior to a series of computations, exploratory runs were made to see if instability was to be anticipated and to compare results from a finer $X$ grid with those of coarser grids. The ranges of initial grid sizes and the multipliers for a sample of volumetric flow rates studied are presented in Table $A 1$. 
TABLE A1.

\begin{tabular}{llll}
\hline$Q$ & $\Delta X$ & $\Delta Y$ & $M$ \\
\hline 0.03 & $7.0 \times 10^{-8}$ & $2.5 \times 10^{-3}$ & 1.08 \\
0.003 & $7.0 \times 10^{-8}$ & $2.5 \times 10^{-3}$ & 1.03 \\
0.0003 & $2.1 \times 10^{-8}$ & $2.5 \times 10^{-3}$ & 1.002 \\
\hline
\end{tabular}

APPENDIX 2

A typical example of the use of the correlations presented in the results is shown below.

$$
\begin{aligned}
\beta & =0.00313{ }^{\circ} \mathrm{K}^{-1}, \ell=10.0 \mathrm{~m}, b=5.08 \mathrm{~cm} \\
T_{0} & =293^{\circ} \mathrm{K}, T_{W}=313^{\circ} \mathrm{K}, T_{\mathrm{g}}=303^{\circ} \mathrm{K} \\
\nu & =1.495 \times 10^{-5} \mathrm{~m}^{2} \cdot \mathrm{s}^{-1}
\end{aligned}
$$

The necessary dimensionless variables are: $G r=3.6 \times 10^{5}, \theta_{\mathrm{g}}=.5$, $\mathrm{L}=5.47 \times 10^{-4}$

then using eqn (34), Q $=0.00970$; from (32),

$$
\overline{N u}_{t}=5.15 ; \text { from }(31), H_{L}=0.00402 ;
$$

from $(15), \theta_{f}=0.415$.

Using the relations in Nomenclature, the dimensionless values may be re-expressed in dimensional form.

$$
\begin{aligned}
\dot{h}_{\ell} & =0.528 \mathrm{kj} \cdot \mathrm{m}^{-1} \cdot \mathrm{s}^{-1} \text { or } 1900 \mathrm{kj} \cdot \mathrm{hr}^{-1} \cdot \mathrm{m}^{-1} \\
q & =0.0522 \mathrm{~m}^{3} \mathrm{~s}^{-1}, \bar{T}=301^{\circ} \mathrm{K} .
\end{aligned}
$$




\section{REFERENCES}

1. F. Trombe, J.F. Robert, M. Cabanat, B. Sesolis. Some performance characteristics of the CNRS solar house collectors, Passive Solar Heating and Cooling, Conference and Workshop Proceedings May 18-19, 1976. Albuquerque, NM, Los Alamos Scientific Laboratory of University of California, Los Alamos, NM 87545, pp. 201-222.

2. J.D. Balcomb, J.C. Hedstrom, R.D. McFarland, Simulation as a design tool, Passive Solar Heating and Cooling, Conference and Workshop Proceedings May 18-19, 1976. Albuquerque, NM, Los Alamos Scientific Laboratory of University of California, Los Alamos, NM 87545, pp. 238-246.

3. W. Elenbaas, Heat dissipation of parallel plates by free convection, Physica 9, 1 (1942).

4. A. Kobayashi, Y. Fujimoto, Study of heat dissipation by natural convection between parallel vertical plates, Trans. Japan Soc. Mech. Engrs. 20, 233 (1954).

5. R. Seige1, R.H. Norris, Tests of free convection in a partially enclosed space between two heated vertical plates, Trans. ASME 79, $663(1957)$.

6. I.G. Currie, W.A. Newman, Natural convection between isothermal vertical surfaces, preprints 4th Int. Heat Transfer Conf., Versailles, Vol. 4, NC 2.7 (1970).

7. T. Aihara, Heat transfer due to natural convection from parallel vertical plates, Trans. Japan Soc. Mech. Engrs. 29 (963), pp. 903.

8. J.R. Bodoia, J.F. Osterle, The development of free convection between heated vertical plates, J. Heat Transfer, Trans. ASME, Ser. C 84, 40 (1962).

9. R.K. Eugel, W.K. Mueller, An analytical investigation of natural convection in vertical channels, ASME paper No. 67-HT-16, (1967).

10. 0. Miyatake, T. Fujii, Free convective heat transfer between vertical parallel plates--one plate is isothermally heated and the other is thermally insulated, KAGAKO KOGAKU, SOc. Chem. Engrs. Japan 36, 405 (1972).

11. T. Aihara, Effects of inlet boundary-conditions on numerical solutions of free convection between vertical parallel plates, Rept. Inst. High Speed Mech., Tohaka Univ., Vol. 28 (1973), pp. 1-27.

12. W.M. Rohsenow, H.Y. Choi, Heat, Mass and Momentum Transfer (Prentice-Hal1, Inc. 1961). 


\section{NOMENCLATURE}

$$
\begin{aligned}
& \text { b = width of channel (plate separation), } m \\
& \beta \quad=\text { coefficient of volumetric thermal expansion, }{ }^{\circ} K^{-1} \\
& C_{p} \quad=\text { specific heat of fluid } \mathrm{kj} \cdot \mathrm{kg}^{-1} \cdot{ }^{\circ} \mathrm{K}^{-1} \\
& \mathrm{~g} \quad=\text { gravitational acceleration, } 9.801 \mathrm{~m} \cdot \mathrm{s}^{-2} \\
& \text { Gr } \quad=\text { Grashof number } g \cdot \beta \cdot\left(T_{m}-T_{0}\right) \cdot b^{3} \cdot \nu^{-2} \\
& \bar{h}=\text { average heat transfer coefficient, } \mathrm{kj} \cdot \mathrm{m}^{-2} \cdot{ }^{\circ} \mathrm{K}^{-1} \cdot \mathrm{s}^{-1} \\
& \dot{h}=\text { rate of heat absorption per } m \text { width of wall, } \mathrm{kj} \cdot \mathrm{m}^{-1} \cdot \mathrm{s}^{-1} \\
& \mathrm{H}_{\mathrm{L}} \quad \text { = dimensionless rate of heat absorption by fluid at channel exit } \\
& \mathrm{H}_{\mathrm{X}} \quad=\text { dimensionless rate of heat absorption by fluid at elevation } X \\
& h_{x, i}=1 \text { local heat transfer coefficient, } k j \cdot m^{-2} \cdot{ }^{\circ} K^{-1} \cdot s^{-1}, i=g, w, t \\
& k=\text { thermal conductivity of fluid, } \mathrm{kj} \cdot \mathrm{m}^{-1} \cdot{ }^{\circ} \mathrm{K}^{-1} \cdot \mathrm{s} \\
& \ell \quad \text { ' }=\text { height of channe1, } m \\
& \mathrm{~L}=\text { dimensionless height of channel } \\
& v \quad=\text { kinematic viscosity } \mathrm{m}^{2} \cdot \mathrm{s}^{-1} \\
& \overline{N u}_{t} \quad=\text { total average Nusselt number } \\
& N u_{x, i}=\text { local Nusselt number, } i=g, w, t \text {, defined by Eqn (16) } \\
& \mathrm{p} \quad=\text { pressure at elevation } \mathrm{x}, \mathrm{kg} \cdot \mathrm{m}^{-2} \\
& \mathrm{p}_{\infty} \quad=\text { ambient pressure at elevation } \mathrm{x}, \mathrm{kg} \cdot \mathrm{m}^{-2} \\
& \mathrm{P}=\text { dimensionless pressure defect } b^{2} \cdot\left(p-p_{\infty}\right) \nu^{-2} \cdot G r^{-2} \cdot p^{-1} \\
& \operatorname{Pr} \quad=\text { Prandt } 1 \text { number } \\
& \mathrm{q} \quad=\text { volume flow rate } \mathrm{m}^{3} \cdot \mathrm{m}^{-1} \cdot \mathrm{s}^{-1} \\
& \text { Q = dimensionless volume flow rate defined by Eqn (13) } \\
& \mathrm{p} \quad=\text { fluid density } \mathrm{kg} \cdot \mathrm{m}^{-3} \\
& \mathrm{~T}=\text { temperature, }{ }^{\circ} \mathrm{K}
\end{aligned}
$$




$$
\begin{aligned}
& \overline{\mathrm{T}} \quad=\text { mixed mean temperature of fluid, }{ }^{\circ} \mathrm{K} \\
& \theta=\text { dimensionless temperature, }\left(T-T_{0}\right) /\left(T_{m}-T_{0}\right) \\
& \bar{\theta}=\text { dimensionless mixed-mean temperature } \\
& \mathrm{T}_{\mathrm{m}} \quad=\text { reference temperature, defined as the temperature of the } \\
& \text { warmer surface of the channe1, }{ }^{\circ} \mathrm{K} \\
& u=\text { fluid velocity, } x \text {-direction, } m \cdot \mathrm{s}^{-1} \\
& \bar{u} \quad=\text { average fluid velocity, } x \text {-direction, } m \cdot s^{-1} \\
& \mathrm{U}=\text { dimensionless fluid velocity, } X \text {-direction } \\
& v=\text { fluid velocity, } y \text {-direction, } \mathrm{m} \cdot \mathrm{s}^{-1} \\
& V=\text { dimensionless fluid velocity, Y-direction } \\
& x, y=\text { cartesian coordinates, } m \\
& X, Y=\text { dimensionless cartesian coordinates }
\end{aligned}
$$

\section{Subscripts}

$$
\begin{array}{ll}
f & =\text { fluid } \\
g & =\text { glass } \\
m & =\text { maximum } \\
0 & =\text { inlet } \\
t & =\text { total } \\
w & =\text { wall } \\
x & =\text { elevation }
\end{array}
$$

The following relations exist:

$$
\begin{aligned}
u & =u \cdot v \cdot G r \cdot b^{-1} \\
l & =L \cdot G r \cdot b \\
v & =v \cdot v \cdot b^{-1} \\
y & =Y \cdot b
\end{aligned}
$$




$$
\begin{array}{ll}
T & =\theta \cdot\left(T_{m}-T_{0}\right)+T_{0} \\
\bar{T} & =\bar{\theta}\left(T_{m}-T_{0}\right)+T_{0} \\
\left(p-p_{\infty}\right) & =p \cdot \rho \cdot v^{2} \cdot G r^{2} \cdot b^{-2} \\
G r & =g \cdot \beta\left(T_{m}-T_{0}\right) \cdot b^{3} \cdot v^{-2} \\
h_{x, g} & =N u_{x, g} \cdot k \cdot b^{-1} \\
h_{x, w} & =N u_{x, w} \cdot k \cdot b^{-1} \\
\bar{h} & =\overline{N u}_{t} \cdot k \cdot b^{-1} \\
\dot{h}_{x} & =H_{x} \cdot v \cdot G r\left(T_{m}-T_{0}\right) \rho \cdot C p \\
H_{L} & =L \cdot \overline{N u}_{t} \cdot \operatorname{Pr}-1 \\
q & =Q \cdot v \cdot G r
\end{array}
$$




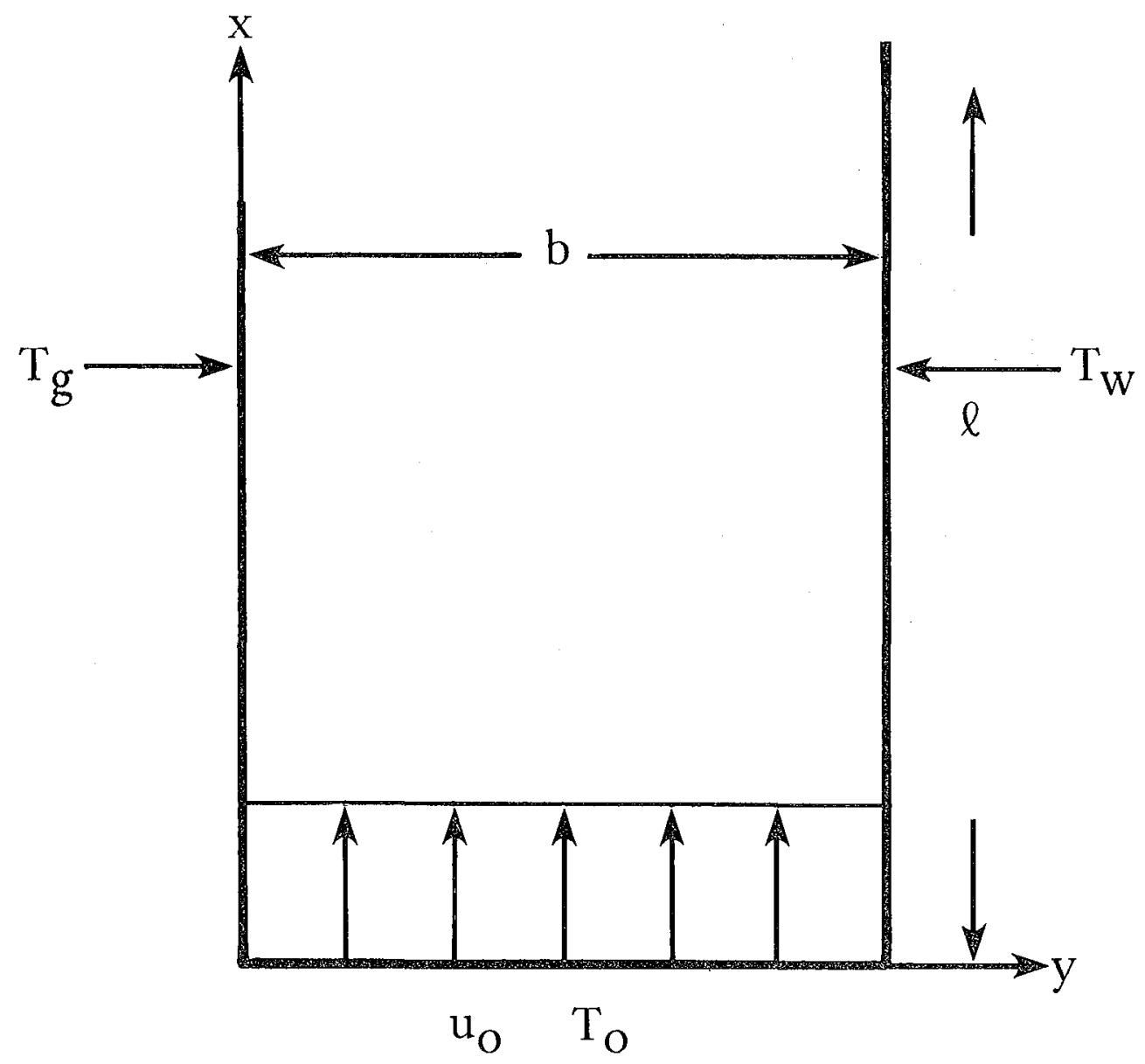

Figure 1. Geometry of flow problem.

XBL 789-10922 


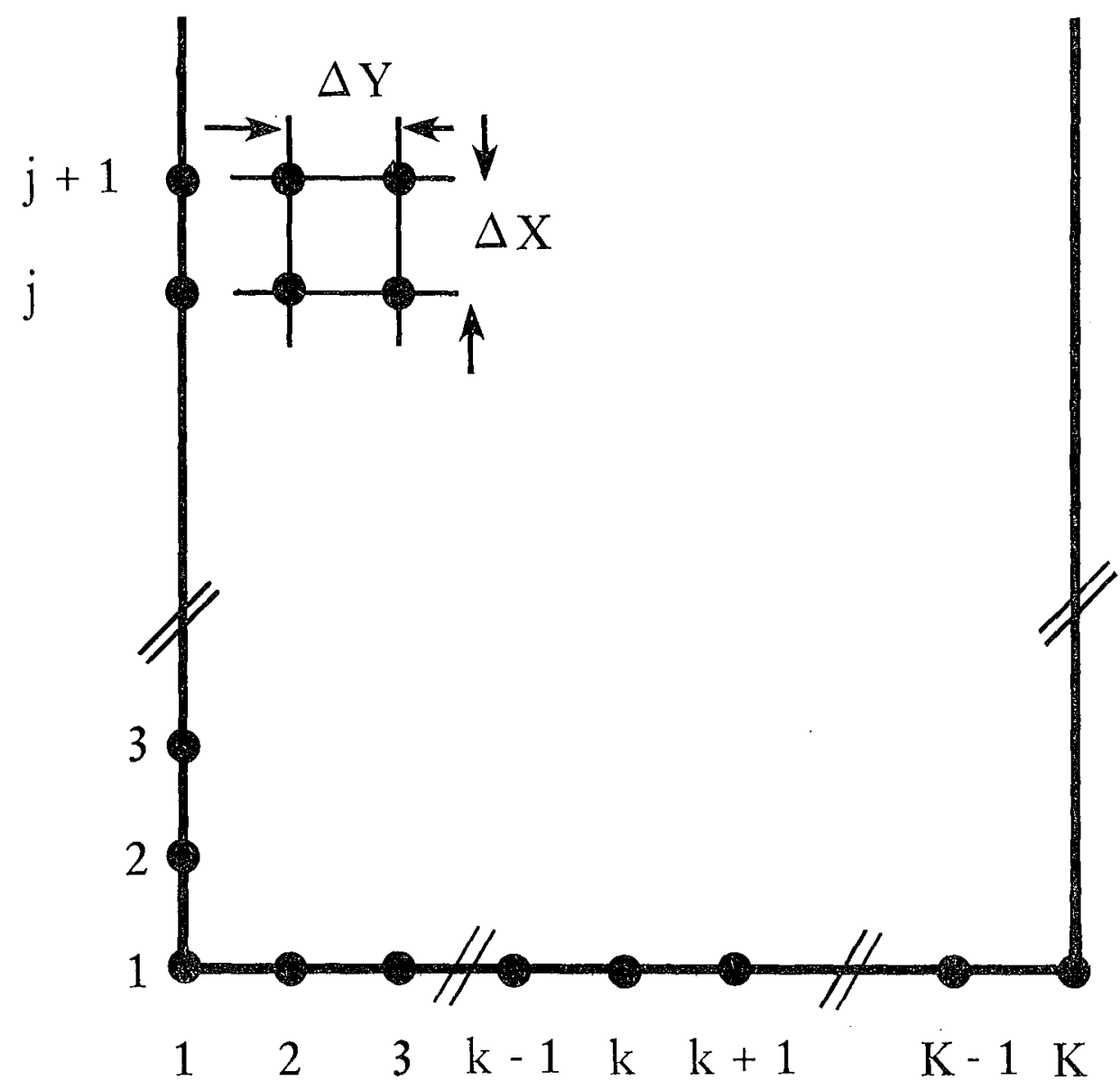

Figure 2. Grid for difference representations.

XBL 784-8216A 

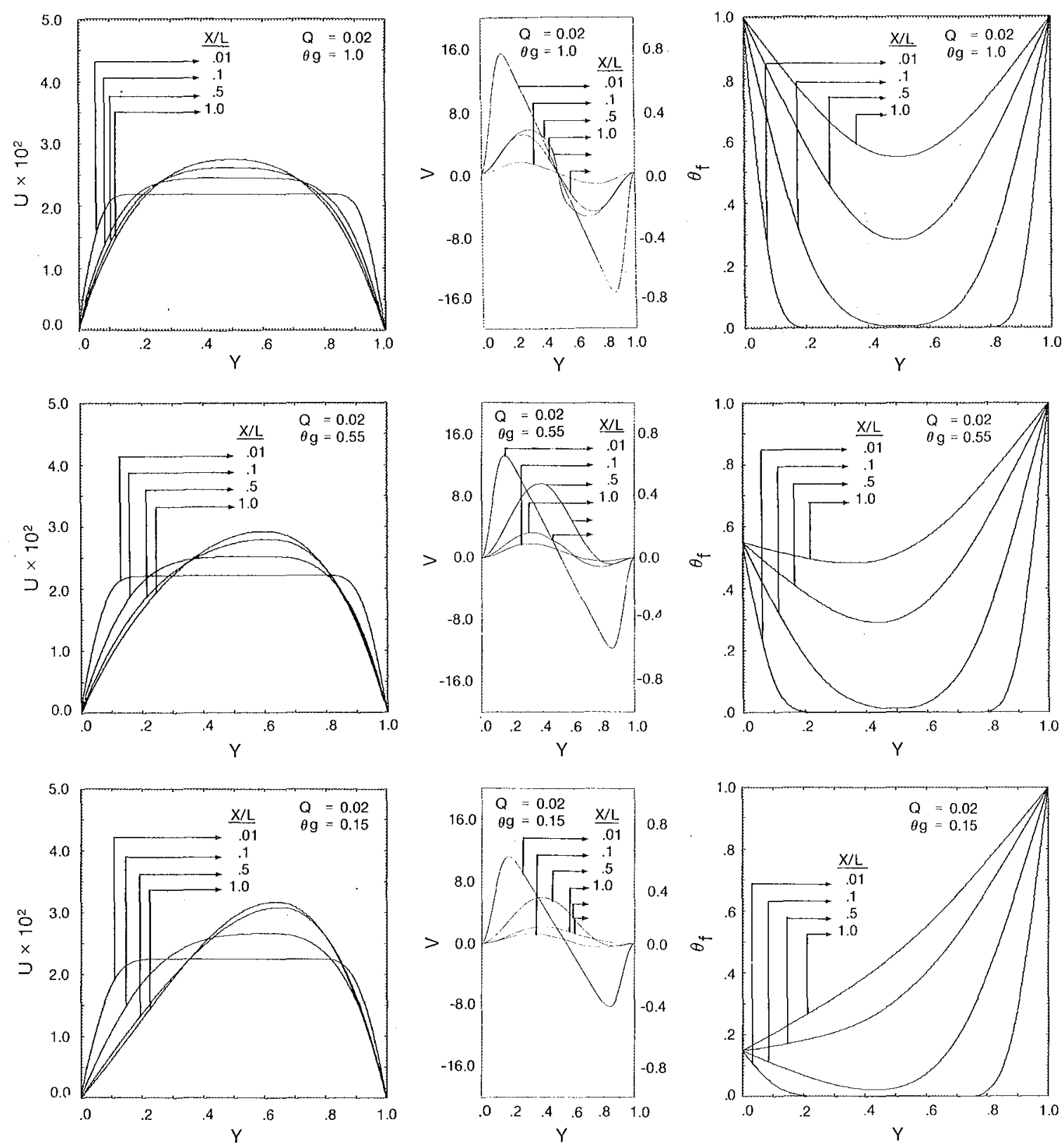

XBL 784-8208

Figure 3. U, V velocity and temperature profiles at four stages of development for three different glass temperatures, $Q=$ 0.02 . 

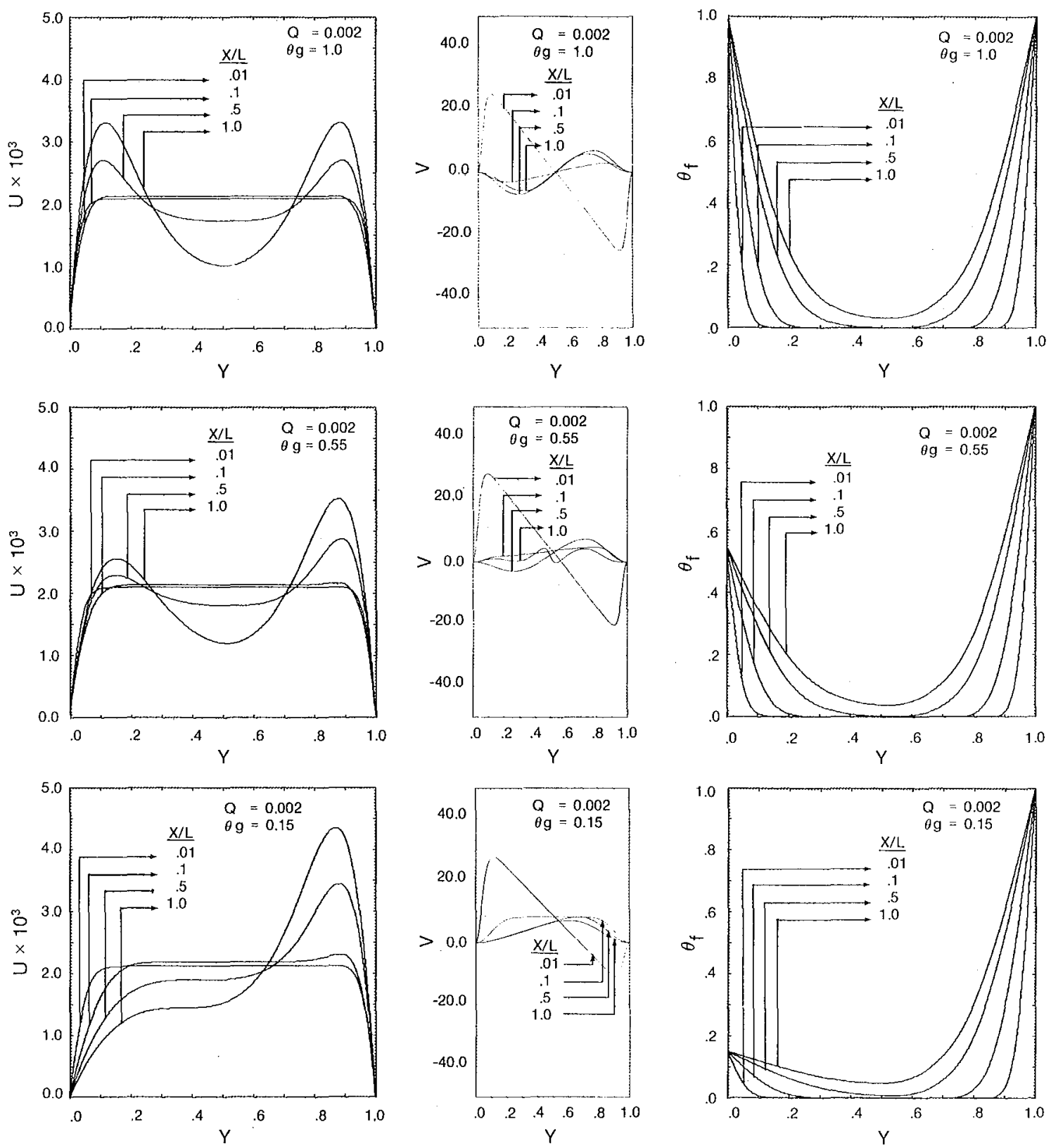

XBL 784-8207

Figure 4. U,V velocity and temperature profiles at four stages of development for three different glass temperatures, $Q=$ 0.002 . 

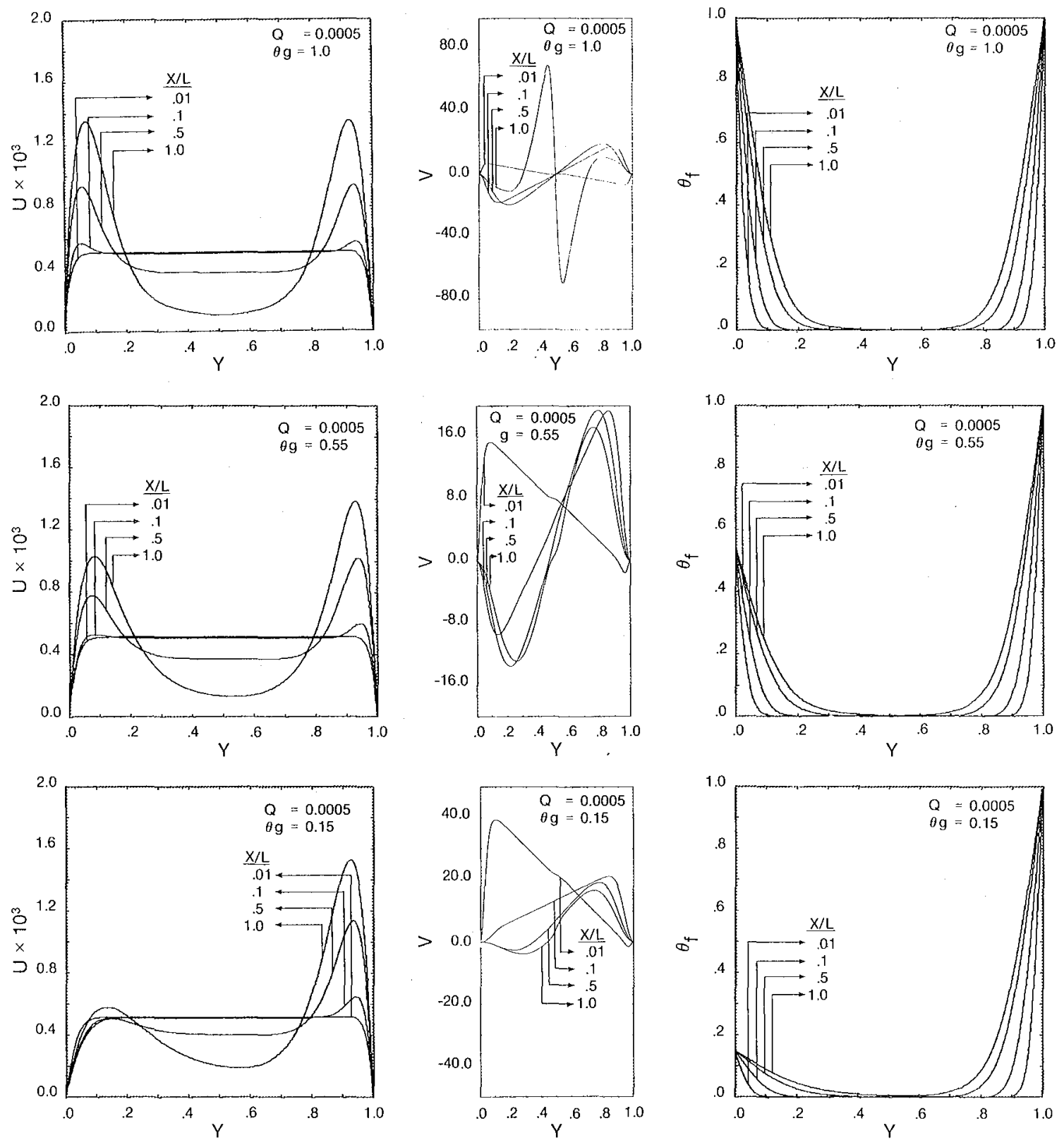

XBL 784-8209

Figure 5. U,V velocity and temperature profiles at four stages of development for three different glass temperatures, $Q=$ 0.0005 . 

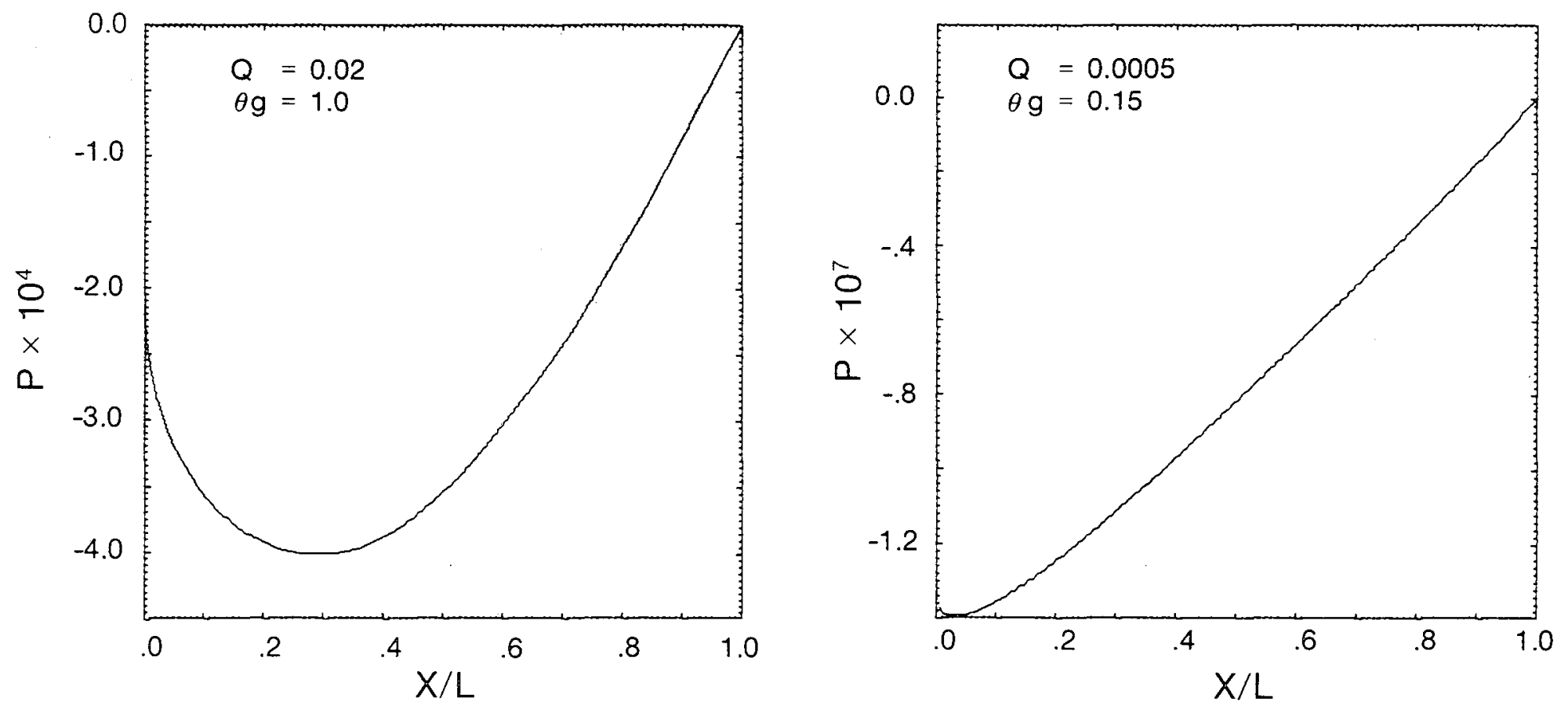

Figure 6. Pressure defect as a function of height for high and low rates. 

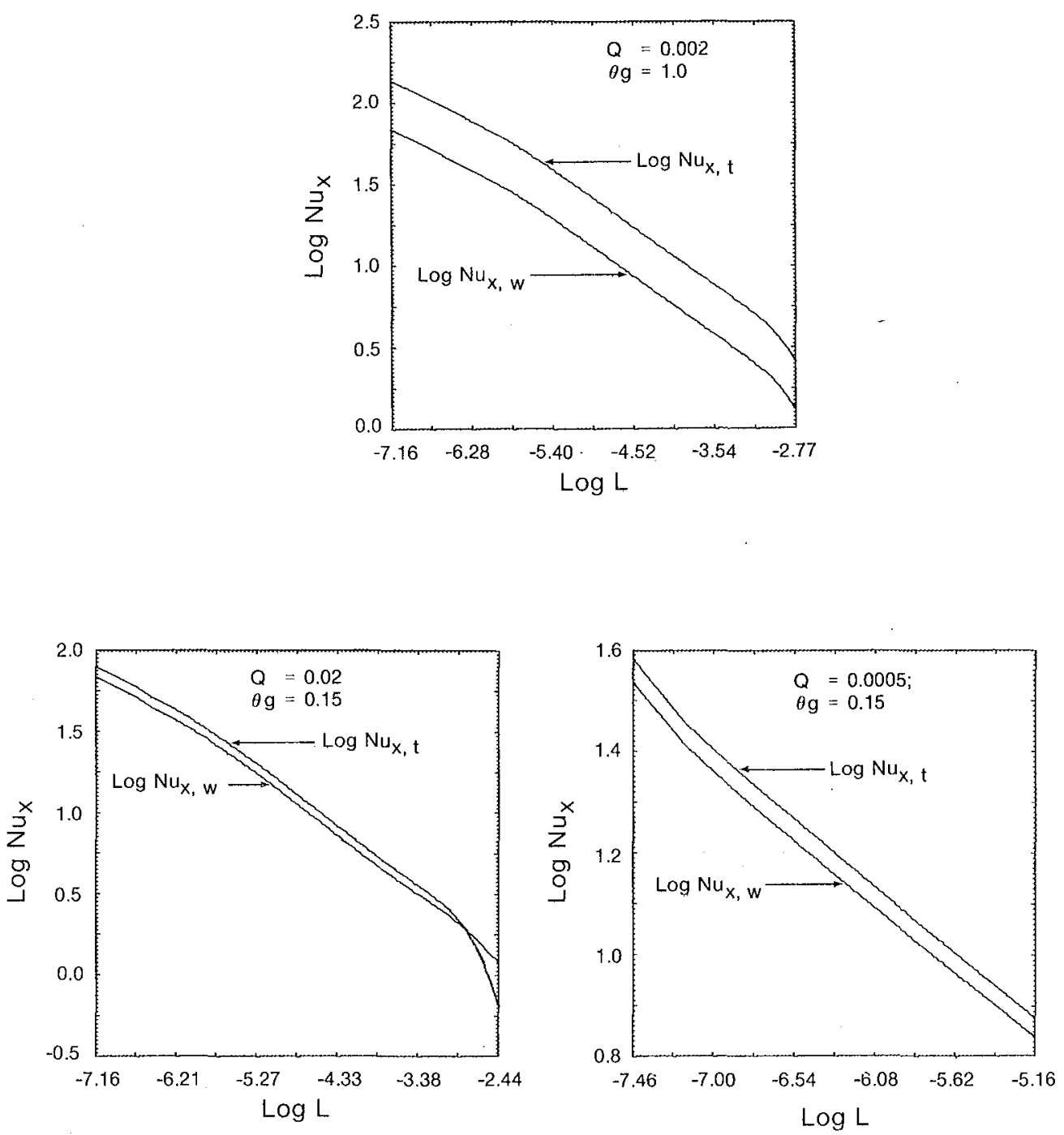

-- XBL 784-8206

Figure 7. The contribution of wall to total local Nusselt number as a function of height for high and low flow rates. 

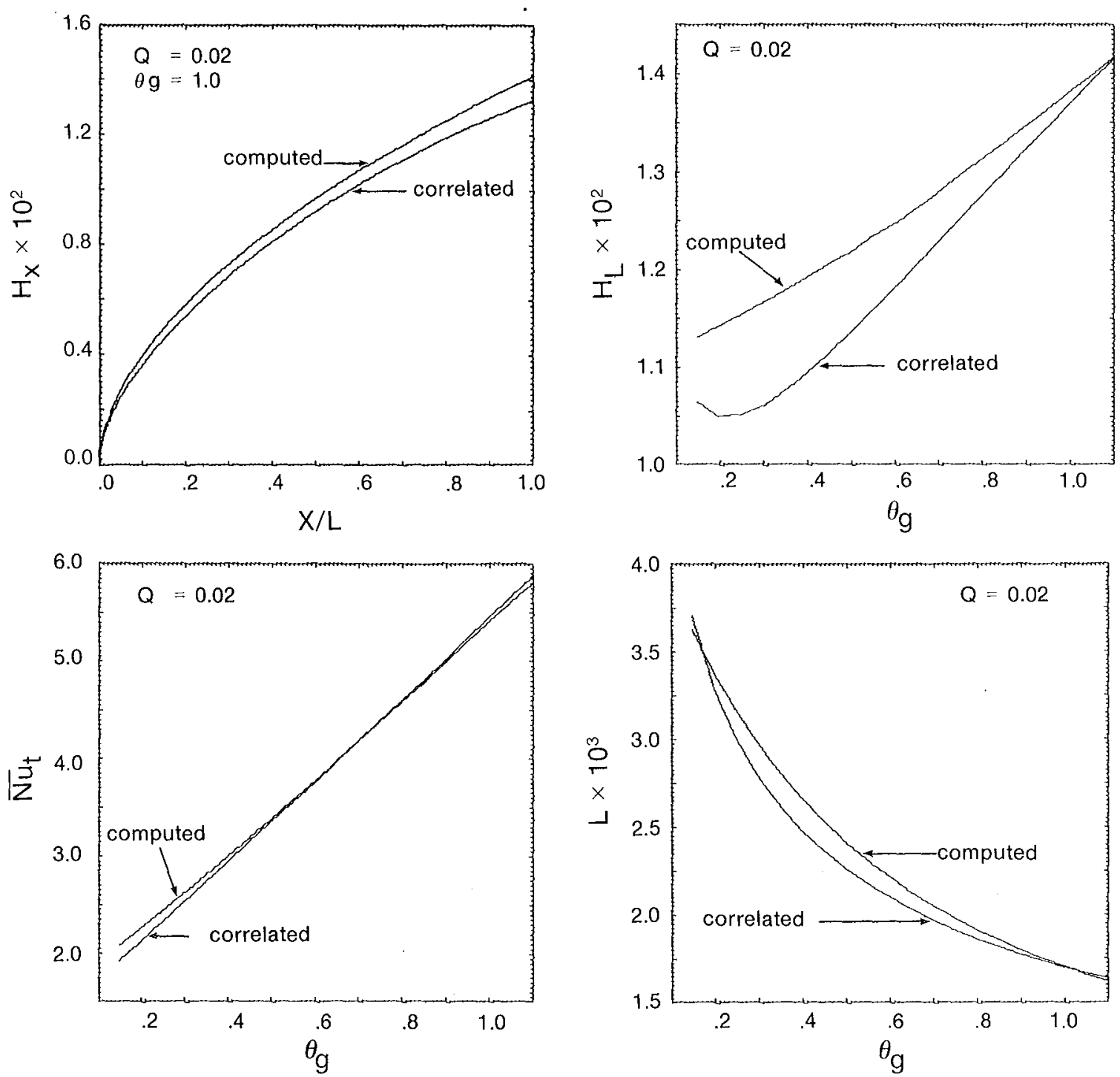

XBL 784-8210

Figure 8. Comparison of computed and correlated results for total heat extracted as a function of height, and the total heat extracted at the exit, the total average Nusselt number, and the total channel height as a function of the glass temperature. 
This report was done with support from the Department of Energy. Any conclusions or opinions expressed in this report represent solely those of the author(s) and not necessarily those of The Regents of the University of California, the Lawrence Berkeley Laboratory or the Department of Energy. 
TECHNICAL INFORMATION DEPARTMENT

LAWRENCE BERKELEY LABORATORY

UNIVERSITY OF CALIFORNIA

BERKELEY, CALIFORNIA 94720 